\title{
Existence of subsonic plasma sheaths
}

\author{
Joaquim Loizu, Paolo Ricci, and Christian Theiler \\ Centre de Recherches en Physique des Plasmas-École Polytechnique Fédérale de Lausanne, \\ Association EURATOM-Confédération Suisse, CH-1015 Lausanne, Switzerland
}

(Received 21 July 2010; revised manuscript received 9 December 2010; published 27 January 2011)

\begin{abstract}
The location of the plasma sheath edge, where quasineutrality is broken, is rigorously derived by using a kinetic description of the plasma. It is shown that sheaths can exist with arbitrarily small ion velocity at the sheath edge, thus violating the Bohm criterion, $V_{i}=c_{s}$ at the sheath edge. Bohm's criterion is recovered in the case of large enough ion current through the wall, and it is found to be a reasonable approximation in floating potential conditions. However, in the case of a predominant electron current through the wall, Bohm's criterion is not able to describe the sheath-edge transition. The analytical results are supported by numerical simulations performed with a fully kinetic particle-in-cell code modeling a source-driven, weakly collisional plasma, bound between two absorbing walls.
\end{abstract}

DOI: 10.1103/PhysRevE.83.016406

PACS number(s): 52.40.Kh, 52.65.Rr

\section{INTRODUCTION}

When a plasma interacts with an absorbing wall, a nonneutral sheath forms. Inside this sheath the electrostatic potential drops on a scale length of the order of the Debye length $\lambda_{D}$, resulting in a large electric field shielding the presheath plasma, which remains quasineutral. In the presheath, the potential varies on a scale length $L \gg \lambda_{D}$, which depends on the physical processes present in this region (e.g., plasma source, ionization, or collisions). Plasma properties at the sheath edge define the boundary conditions for any model assuming quasineutrality; hence their knowledge is essential to describe the dynamics of the main plasma and to predict the particle and energy fluxes at the solid surface. Sheaths have been studied since the pioneering work of Langmuir in 1929 [1], followed by an enormous research effort [2-4], which persists still (see, e.g., Refs. [5-9]). Only recently has their detailed experimental investigation become possible [10-12]. Sheaths are present in the edge of magnetically confined fusion plasmas, at the interface between spacecrafts and space plasmas, in the fabrication of semiconductor devices, and wherever a plasma interacts with a solid surface.

The commonly accepted theory to describe the sheath-edge location is the Bohm criterion, stating that ions need to be accelerated up to the plasma sound speed $c_{s}=\sqrt{T_{e} / m_{i}}$ in order for a sheath to exist [13]. One can derive Bohm's criterion assuming that electrons have a constant temperature $T_{e}$ and follow the Boltzmann relation $n_{e}=n_{\mathrm{se}} \exp \left[e\left(\phi-\phi_{\mathrm{se}}\right) / T_{e}\right]$, where $n_{\text {se }}$ and $\phi_{\text {se }}$ denote the electron density and the plasma potential at the sheath edge. Ions are assumed to be monoenergetic $\left(T_{i}=0\right)$ and collisionless, with a velocity $v_{\infty}=0$ far from the sheath. The conservation of ion flux, $\Gamma_{i}=n_{i} V_{i}$, and energy, $m_{i} V_{i}^{2} / 2=e\left(\phi_{\infty}-\phi\right)$, where $\phi_{\infty}$ is the main plasma potential, leads to estimating the ion density as $n_{i}=n_{\mathrm{se}} \sqrt{\Delta \phi_{\mathrm{ps}} /\left(\phi_{\infty}-\phi\right)}$. Here $\Delta \phi_{\mathrm{ps}}=\phi_{\infty}-\phi_{\mathrm{se}}$ is the presheath potential drop. Bohm's criterion is obtained by linearizing Poisson's equation around $\phi=\phi_{\mathrm{se}}$,

$$
\frac{\partial^{2} \phi}{\partial x^{2}} \approx e^{2} n_{\mathrm{se}}\left(\frac{1}{T_{e}}-\frac{1}{2 e \Delta \phi_{\mathrm{ps}}}\right)\left(\phi-\phi_{\mathrm{se}}\right)
$$

and showing that $V_{i, \text { se }} \geqslant c_{s}$, since a physically acceptable, nonoscillatory solution for $\phi$ exists only if $\partial_{x}^{2} \phi \leqslant 0$ [2]. Therefore ions are at least sonic when entering the sheath. On the other hand, by treating separately the presheath region, one can show that $V_{i} \leqslant c_{s}$ before entering the sheath [3], thus implying that the sheath-edge location is defined by the point where $V_{i}=c_{s}$. The possibility of the existence of sheaths with subsonic ions at the edge was widely addressed in the literature, by exploring if the presheath processes (collisions, ionization) or the geometry could influence the transition to the non-neutral region $[14,15]$. It was concluded that the Bohm criterion, $V_{i}=c_{s}$, is a ubiquitous property of ion sheaths in the limit $T_{i} \ll T_{e}$ and $\lambda_{D} \ll L[2,16]$.

In this article we rigorously derive the location of the sheath edge where quasineutrality is broken by using an appropriate kinetic description of the plasma. We show that an ion sheath can exist with arbitrarily small ion velocity and that this may be relevant in many situations. Bohm's criterion is recovered in the case of a large enough ion current through the wall $\left(\Gamma_{i} \gg \Gamma_{e}\right)$, and it is found to be a reasonable approximation in floating potential conditions $\left(\Gamma_{i}=\Gamma_{e}\right)$. However, in the case of a predominant electron current through the wall $\left(\Gamma_{e}>\Gamma_{i}\right)$, Bohm's criterion is not able to describe the sheath-edge transition. The general assumptions of our work are a very small ion to electron temperature ratio, $T_{i} \ll T_{e}$, weakly collisional electrons with a mean free path larger than the sheath scale, $\lambda_{\operatorname{mfp}} \gg \lambda_{D}$, and a totally absorbing wall. Our results are supported by numerical simulations performed with a fully kinetic particle-in-cell (PIC) code modeling a source-driven, weakly collisional plasma, bound between two absorbing walls.

\section{DERIVATION OF THE SHEATH-EDGE LOCATION}

We start by writing the first two moments of Vlasov equation for ions and the first moment for electrons, i.e., 
continuity and momentum equations, which in steady-state conditions are

$$
\begin{gathered}
n_{i} \frac{\partial V_{i}}{\partial x}+V_{i} \frac{\partial n_{i}}{\partial x}=S_{\mathrm{pi}}, \\
n_{e} \frac{\partial V_{e}}{\partial x}+V_{e} \frac{\partial n_{e}}{\partial x}=S_{\mathrm{pe}}, \\
m_{i} n_{i} V_{i} \frac{\partial V_{i}}{\partial x}=-e n_{i} \frac{\partial \phi}{\partial x}+S_{\mathrm{mi}} .
\end{gathered}
$$

The particle and momentum sources, $S_{p}$ and $S_{m}$, result from integrating the terms in the Vlasov equation related to the injection of particles, ionization processes, or collisions. The ion pressure is neglected since $T_{i} \ll T_{e}$. In the following, the potential is defined so that at the wall $\phi_{w}=0$, implying that $\phi(x)$ represents the potential drop up to the wall. We now express $V_{e}$ by using our knowledge of the electron distribution function. In fact, in the case of a monotonic ion sheath, the electron velocity distribution function approaching a wall can be described by a truncated Maxwellian [4,17], as confirmed experimentally $[18,19]$ and by our simulations. The cutoff velocity is due to the fact that all the electrons having an energy above the potential barrier flow out from the system, and no electrons can be reflected with $v_{x}>\sqrt{2 e \phi(x) / m_{e}}=v_{\text {cut }}$. By defining the quantity $\eta(x)=e \phi(x) / T_{b}$, where $T_{b}$ is the bulk temperature in the main plasma, we have

$$
f_{e}\left(v_{x}, \eta\right)= \begin{cases}\frac{1}{I(\eta) \sqrt{2 \pi v_{\text {thb }}^{2}}} e^{-\frac{v_{x}^{2}}{2 v_{\text {thb }}^{2}}} & \text { if } v_{x}<v_{\text {cut }}(\eta) \\ 0 & \text { otherwise }\end{cases}
$$

where $v_{\text {thb }}=\sqrt{T_{b} / m_{e}}, v_{\text {cut }}(\eta)=\sqrt{2 \eta} v_{\text {thb }}$ is the cutoff velocity, and $I(\eta)=[1+\operatorname{erf}(\sqrt{\eta})] / 2$ is the normalization factor. We can now compute the electron fluid velocity $V_{e}=\left\langle v_{x}\right\rangle$, having defined $\langle a\rangle=\int f_{e}\left(v_{x}\right) a\left(v_{x}\right) d v_{x} . V_{e}$ increases as the Maxwellian is progressively truncated when approaching the wall,

$$
V_{e}=\frac{c_{\mathrm{sb}}}{I(\eta)} e^{\Lambda-\eta}
$$

where $c_{\mathrm{sb}}=\sqrt{T_{b} / m_{i}}, \quad \Lambda=\log \sqrt{\mu / 2 \pi}$ and $\mu=m_{i} / m_{e}$. Equation (4) is the common expression used as a sheath boundary condition except for the correction given by $I(\eta)$. We also note that the term $\partial_{x} V_{e}$ in system (2) can be evaluated as $\partial_{x} V_{e}=$ $\partial_{\phi} V_{e} \partial_{x} \phi$, where $\partial_{\phi} V_{e}=-\left(e V_{e} / T_{b}\right)\left[1+e^{-\eta} / 2 \sqrt{\pi \eta} I(\eta)\right]$.

Thus far the system (2) together with Eq. (4) is very general and should be satisfied within both the sheath and the presheath regions, as long as the collisionality is small enough for the closure to be valid. In the presheath, quasineutrality is preserved, and the condition $n_{e}=n_{i}=n$ has to be fulfilled up to the sheath entrance. By imposing it, we are left with three unknowns $\left(n, V_{i}, \phi\right)$ and their respective gradients, and our system of equations can be reduced to a matrix system M $\vec{X}=\vec{S}$, where

$$
\vec{X}=\left(\begin{array}{c}
\partial_{x} n \\
\partial_{x} V_{i} \\
\partial_{x} \phi
\end{array}\right), \quad \vec{S}=\left(\begin{array}{c}
S_{\mathrm{pi}} \\
S_{\mathrm{pe}} \\
S_{\mathrm{mi}}
\end{array}\right)
$$

and the matrix $\mathbf{M}$ depends only on local quantities,

$$
\mathbf{M}=\left(\begin{array}{ccc}
V_{i} & n & 0 \\
V_{e} & 0 & n \partial_{\phi} V_{e} \\
0 & m_{i} n V_{i} & e n
\end{array}\right) .
$$

This reduced system is valid in the presheath up to the sheath edge. In the presheath region, gradients are typically small and are due to the presence of the plasma source. At the sheath edge, gradients become much steeper, i.e., $\left|M_{\alpha \beta} X_{\beta}\right| \gg\left|S_{\alpha}\right|$ for all $\alpha, \beta$ such that $M_{\alpha \beta} \neq 0$. In other words, at the sheath edge the source terms are much smaller than any other term in the fluid equations, and the fluid system (2) reduces to $\mathbf{M} \vec{X} \simeq 0$. Now, the presence of nonzero gradients imposes $\operatorname{det}(\mathbf{M})=0$, which defines the position of the sheath edge. We note that $\operatorname{det}(\mathbf{M})=0$ is also a valid definition of the sheath edge in the particular case of a source-free system. In fact, in this case $\mathbf{M} \vec{X}=0$ is satisfied everywhere in the presheath, and the macroscopic quantities display flat profiles [20]; therefore $\vec{X}=0$. At the sheath edge, gradients become nonzero, still requiring $\operatorname{det}(\mathbf{M})=0$. Hence in all cases $\operatorname{det}(\mathbf{M})=0$ at the sheath entrance, which gives

$$
V_{i, \mathrm{se}}=c_{\mathrm{sb}} \sqrt{\frac{1}{1+\kappa}},
$$

where

$$
\kappa=\frac{e^{-\eta_{\mathrm{se}}}}{2 \sqrt{\pi \eta_{\mathrm{se}}} I\left(\eta_{\mathrm{se}}\right)} .
$$

Figure 1 shows the dependence of $V_{i, \text { se }}$ and $V_{e \text {,se }}$ on the sheath-edge potential $\eta_{\mathrm{se}}$. The condition of ambipolar flow $\Gamma_{i}=\Gamma_{e}$ can be found by solving $V_{i, \mathrm{se}}=V_{e, \text { se }}$, expressed by Eqs. (4) and (7). This defines the floating potential, which is found to be at $\eta_{\text {se }} \approx \Lambda$ for the hydrogen mass ratio $(\mu=1836$, $\Lambda \simeq 2.8$ ) or higher. In correspondence to the floating potential, we have $V_{i, \mathrm{se}} \approx c_{\mathrm{sb}}$. In the limit of $\eta_{\mathrm{se}} \rightarrow \infty$, or equivalently $\Gamma_{i} \gg \Gamma_{e}$, one has that $\kappa \rightarrow 0$, thus reducing Eq. (7) to Bohm's criterion. However, for $\eta_{\mathrm{se}} \rightarrow 0$, or equivalently $\Gamma_{e} \gg \Gamma_{i}$, the function $\kappa \rightarrow \infty$ and the ion velocity $V_{i, \text { se }} \rightarrow 0$. From this we conclude that Bohm's criterion is not valid in general, since it is violated when $\eta_{\mathrm{se}}<\Lambda$ (or $\left.\Gamma_{e}>\Gamma_{i}\right)$.

\section{SIMULATION RESULTS}

In order to confirm the validity of the presented results, we perform numerical simulations with a fully kinetic PIC code, akin to previous simulations [20,21]. The system size is much larger than the sheath scale $\left(L \simeq 10^{3} \lambda_{D}\right)$. A source of ions and electrons uniformly distributed between two absorbing walls maintains the plasma in steady state. Ions have a temperature much smaller than the electrons $\left(T_{i} / T_{e} \simeq 10^{-2}\right)$, electrons undergo elastic collisions with each other according to a Fokker-Plank collision operator [22], with a mean free path much larger than the sheath scale $\left(\lambda_{\text {mfp }} \simeq 300 \lambda_{D}\right)$, and Poisson's equation is solved by imposing a fixed potential at the two boundaries, $\phi_{w}=0$. The mass ratio is $\mu=400$ (essentially the same results are obtained in tests performed with $\mu=800)$. Sheath currents can be driven by injecting an unbalanced amount of ions and electrons throughout the 
(a)

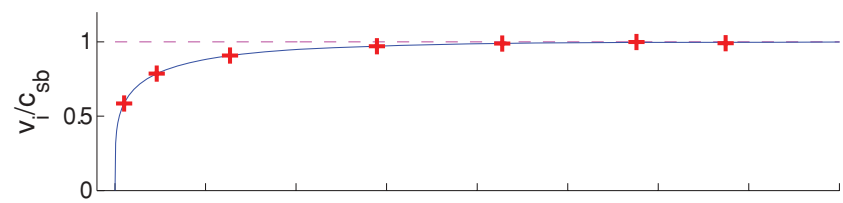

(b)
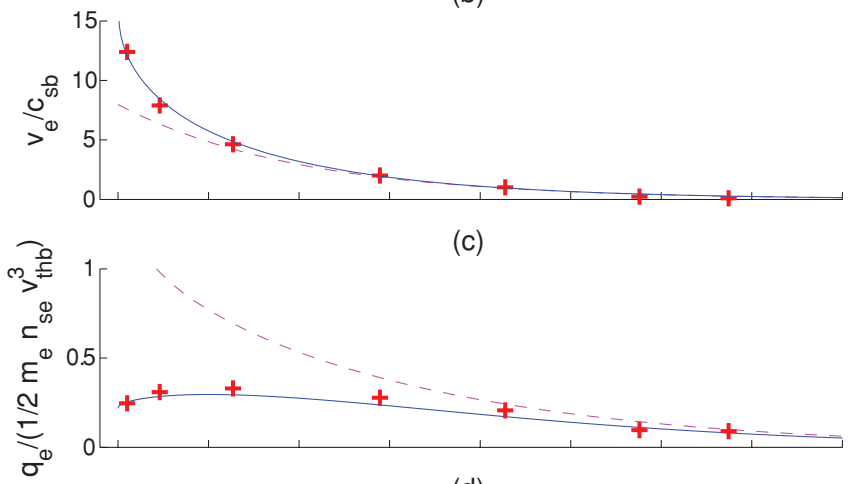

(d)

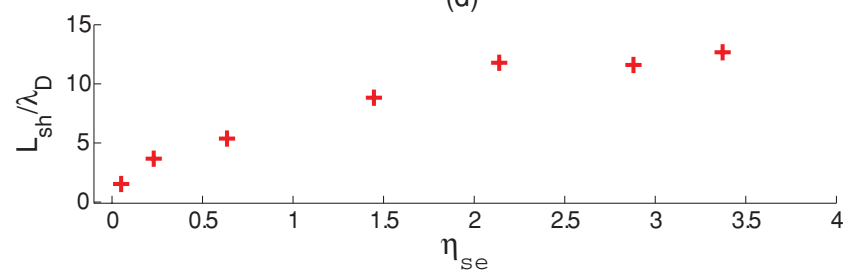

FIG. 1. (Color online) Sheath-edge quantities as a function of the normalized sheath-edge potential $\eta_{\mathrm{se}}$. (a-c) Analytical expressions (solid blue lines) for $V_{i}, V_{e}$, and $q_{e}$ [Eqs. (4), (7), and (9)] are compared with the simulations results (red crosses). The sheath-edge position is found according to Eq. (7). The expressions deduced from textbook formulas are also shown (dashed magenta lines), i.e., $V_{i}=c_{s}, V_{e}=c_{s} \exp (\Lambda-\eta)$, and $q_{e}=Q_{e}-\delta$, where $Q_{e}=$ $\gamma \Gamma_{e} T_{b}$ is the macroscopic heat flux [3], $\gamma=\eta_{\mathrm{se}}+\Delta \phi_{\mathrm{ps}} / T_{b}$, and $\delta=\left(m_{e} n_{\mathrm{se}} / 2\right)\left(\langle v\rangle^{3}+3\langle v\rangle\left\langle(v-\langle v\rangle)^{2}\right\rangle\right)$ is evaluated according to Eq. (3). (d) The sheath length $L_{\mathrm{sh}}$ is obtained from the simulations and normalized to the local Debye length $\lambda_{D}=\sqrt{T_{b} /\left(e^{2} n_{\mathrm{se}}\right)}$. The only expression that depends on the mass ratio is $V_{e} / c_{\mathrm{sb}}$, plotted here for $\mu=400$.

domain, in such a way that the sheath potential is varied and sheath-edge quantities are studied as a function of $\eta_{\mathrm{se}}$.

Simulations confirm that the sheath-edge position is well described by Eq. (7). Figure 2 shows, in fact, that the position of the sheath entrance defined by (7) is always coherent with the breaking of quasineutrality, while Bohm's criterion fails to describe the transition for small values of $\eta_{\mathrm{se}}$. In particular, for $\eta_{\text {se }} \lesssim 0.25$, we observe that $V_{i}<c_{s}$ everywhere, even at the wall, and yet a significant charge imbalance with a smooth potential drop is observed, pointing out the existence of a sheath. The numerical results for $V_{i}, V_{e}$, and the microscopic heat flux, $q_{e}=n_{e} m_{e}\left\langle\left(v_{x}-V_{e}\right)^{3}\right\rangle / 2$, at the sheath edge are shown in Fig. 1 for different values of $\eta_{\text {se }}$ and compared with the analytical predictions provided by Eqs. (4), (7), and

$q_{e}=\frac{n_{e} m_{e} v_{\mathrm{thb}}^{3}}{\sqrt{2 \pi} I(\eta)}\left[e^{-\eta}\left(\eta-\frac{1}{2}\right)+\frac{3}{2} \sqrt{\frac{\eta}{\pi}} \frac{e^{-2 \eta}}{I(\eta)}+\frac{e^{-3 \eta}}{2 \pi I^{2}(\eta)}\right]$ (a)
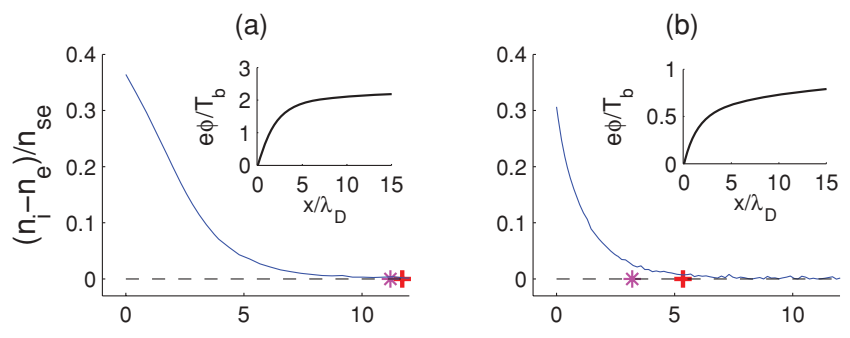

(c)

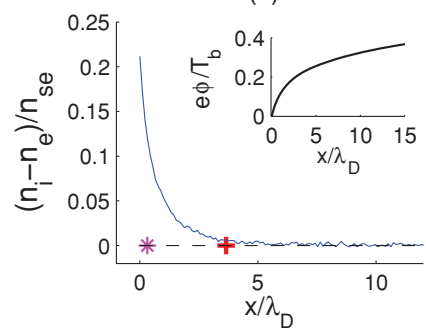

(d)

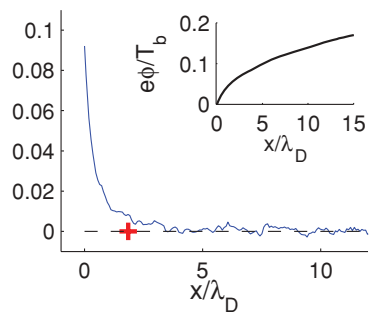

FIG. 2. (Color online) Steady-state charge imbalance in the vicinity of the wall (inset: potential profiles). Results from the simulations are shown for four different cases, with increasing electron current: (a) $\eta_{\mathrm{se}} \approx 2.1$ (floating potential for $\mu=400$ ), (b) $\eta_{\mathrm{se}} \approx 0.63$, (c) $\eta_{\mathrm{se}} \approx 0.22$, (d) $\eta_{\mathrm{se}} \approx 0.05$. The locations of the sheath edge according to Bohm's criterion (magenta stars) and according to Eq. (7) (red crosses) are displayed. In (d) the ion velocity never reaches $c_{s}$ so according to Bohm's criterion there is no sheath.

The results for $V_{e}$ and $q_{e}$ are in addition compared with the corresponding analytical expressions deduced from textbook formulas [3], showing disagreement for $\eta_{\mathrm{se}}<\Lambda$. The sheath length, $L_{\mathrm{sh}}$, is also shown in Fig. 1 as a function of $\eta_{\mathrm{se}}$. The sheath is expanded in the region where $\Gamma_{i}>\Gamma_{e}$, consistent with the Child-Langmuir model [4], whereas it is compressed when $\Gamma_{e}>\Gamma_{i}$, with $L_{\mathrm{sh}} \rightarrow 0$ when $\eta_{\mathrm{se}} \rightarrow 0$.

\section{DISCUSSION AND CONCLUSION}

How can a smooth sheath exist with subsonic ion velocity at the sheath edge and yet $\partial_{x}^{2} \phi<0$, which is impossible according to Eq. (1)? The fact is that Eq. (1) assumes that $n_{e}$ decreases according to the Boltzmann factor, which accounts for the fraction of the electron population that is reflected before reaching the wall. However, the absorbing boundary reduces even more the electron density since it gives rise to a truncated distribution function [4,23]. The more general formula is

$$
n_{e}=n_{\mathrm{se}} \exp \left[\frac{e\left(\phi-\phi_{\mathrm{se}}\right)}{T_{b}}\right] \frac{I(\eta)}{I\left(\eta_{\mathrm{se}}\right)},
$$

which reduces to the Boltzmann relation in the limit of large $\eta_{\text {se }}$. For small values of $\eta_{\text {se }}$, this correction is important, and the linearized Poisson equation with the expression for $n_{e}$ given by Eq. (10) is

$$
\frac{\partial^{2} \phi}{\partial x^{2}} \approx e^{2} n_{\mathrm{se}}\left[\frac{1}{T_{b}}(1+\kappa)-\frac{1}{2 e \Delta \phi_{\mathrm{ps}}}\right]\left(\phi-\phi_{\mathrm{se}}\right) .
$$

If we impose $\partial_{x}^{2} \phi \leqslant 0$ we find $V_{i, \mathrm{se}} \geqslant c_{\mathrm{sb}} \sqrt{1 /(1+\kappa)}$, an inequality that is compatible with Eq. (7), showing that it is therefore possible to find smooth sheath solutions with 
subsonic ions at the sheath entrance. Since the function $\kappa \rightarrow \infty$ as $\eta_{\mathrm{se}} \rightarrow 0$, the scaling analysis of Eq. (11) shows that $L_{\mathrm{sh}} \rightarrow 0$ in this limit, as confirmed by Fig. 1 .

An experimental verification of these predictions could be carried out by measuring the ion velocity in the vicinity of an ion sheath that has been biased so that the plasma potential is only slightly above the wall potential. Such sheaths have been recently produced and characterized, showing monotonic potential profiles [24].

The results presented herein are relevant in a number of physical situations. As an example, we cite here the Edge Localized Modes in tokamak fusion devices, where large plasma currents to the divertor plates can be observed [25,26]. In the case of a transient event where $\Gamma_{e} \gg \Gamma_{i}$, corresponding to small values of $\eta_{\text {se }}$, according to Eq. (7) the ion flow is strongly reduced with respect to Bohm's prediction. This is important because ions determine the plasma momentum flux, even in the case of predominant electron current. We also mention the plasma thrusters used for spacecraft propulsion, where large electron currents are locally observed in the conducting walls [27]. As a last example, we allude to the subsonic origin of the solar wind plasma, which has been recently explained through a gravito-electrostatic sheath created at the surface boundary of the sun. This boundary acts as a negatively biased wall and thus also draws electron current [28]. In general, our results are important for setting the boundary conditions at the sheath edge in plasma fluid models.

We conclude that in floating conditions where $\eta_{\mathrm{se}} \approx \Lambda$, or in the case of predominant ion current $\left(\Gamma_{i}>\Gamma_{e}\right)$, the usual Bohm criterion together with the commonly used expression for the electron velocity is a reasonable approximation.
However, when the plasma sheath potential is small $\left(\Gamma_{e}>\Gamma_{i}\right)$, Bohm's theory is not consistent anymore with the breaking of quasineutrality. The electron kinetic effects have a strong impact on the ion velocity, and the sheath-edge definition needs to be refined according to Eq. (7). This new definition of the sheath edge is coherent with the breaking of neutrality and other sheath-edge quantities and is valid for all $\eta_{\text {se }}>0$. It also applies if there is a magnetic field perpendicular to the wall. Its validity breaks down if the electron mean free path becomes very small, $\lambda_{\text {mfp }} \sim \lambda_{D}$, if the source terms become large enough to strongly affect the properties of the sheath, and for $\eta_{\mathrm{se}}<0$, since in this case Eq. (3) is not valid anymore. Finally, Eq. (7) can also be derived by considering the general dispersion relation of ion-acoustic waves in the limit $\omega / k=0$ [29] with the distribution function given by Eq. (3). In this respect, however, we notice that the method described in the present article provides a new rigorous way of deriving the sheath-edge location, leading to the sheath criterion directly in its equality form, thus avoiding the problem of matching the presheath and sheath regions. Moreover such a technique makes it possible to determine how gradients are related to each other at the sheath edge. In summary, this work points out a correction to the Bohm criterion that shows how sheaths can become subsonic in many relevant physical situations.

\section{ACKNOWLEDGMENTS}

We acknowledge many useful discussions with S. Brunner, A. Fasoli, I. Furno, K. Gustafson, A. Howling, and T.-M. Tran. We also acknowledge financial support by the Fonds National Suisse de la Recherche Scientifique.
[1] I. Langmuir, Phys. Rev. 33, 954 (1929).

[2] K.-U. Riemann, J. Phys. D. 24, 493 (1991).

[3] P. Stangeby, The Plasma Boundary of Magnetic Fusion Devices (IOP Publishing, Bristol, 2000).

[4] I. H. Hutchinson, Principles of Plasma Diagnostics (Cambridge University Press, Cambridge, 2002).

[5] D. Tskhakaya et al., Phys. Plasmas 11, 3945 (2004).

[6] K.-U. Riemann et al., Phys. Plasmas 13, 063508 (2006).

[7] N. Jelic et al., Phys. Plasmas 14, 103506 (2007).

[8] D. Sydorenko, I. Kaganovich, Y. Raitses, and A. Smolyakov, Phys. Rev. Lett. 103, 145004 (2009).

[9] S. D. Baalrud, C. C. Hegna, and J. D. Callen, Phys. Rev. Lett. 103, 205002 (2009).

[10] N. Hershkowitz, Phys. Plasmas 12, 055502 (2005).

[11] L. Oksuz, D. Lee, and N. Hershkowitz, Plasma Sources Sci. Technol. 17(1), 015012 (2008).

[12] T. Lunt, G. Fussmann, and O. Waldmann, Phys. Rev. Lett. 100, 175004 (2008).

[13] D. Bohm, The Characteristics of Electrical Discharges in Magnetic Fields, edited by A. Guthrie and R. K. Wakerling (McGraw-Hill, New York, 1949), p. 77.
[14] L. S. Hall, Phys. Fluids 11, 388 (1961).

[15] Zawaideh et al., Phys. Fluids B 2, 647 (1990).

[16] K.-U. Riemann et al., Phys. Plasmas 4, 4158 (1997).

[17] G. Kawamura et al., Phys. Plasmas 14, 083502 (2007).

[18] S.W. Rayment et al., Proc. Roy. Soc. A. 340, 87 (1968).

[19] A. Maresca, K. Orlov, and U. Kortshagen, Phys. Rev. E 65, 056405 (2002).

[20] J. R. Procassini et al., Phys. Fluids B 2, 3191 (1990).

[21] J. R. Procassini et al., Phys. Fluids B 3, 1876 (1991).

[22] T. Takizuka, J. Comput. Phys. 25, 205 (1977).

[23] J. Igold, Phys. Fluids 15, 75 (1972).

[24] S. D. Baalrud et al., Phys. Plasmas 14, 042109 (2007).

[25] T. D. Rognlien et al., J. Nucl. Mater. 313-316, 1000 (2003).

[26] M. E. Fenstermacher et al., Plasma Phys. Control. Fusion 45, 1597 (2003).

[27] S. Barral et al., Phys. Plasmas 10, 4137 (2003).

[28] C. B. Dwivedi et al., Astrophys. J. 663, 1340 (2007).

[29] J. E. Allen, Plasma Sources Sci. Technol. 18, 014004 (2009). 